\title{
Modelo para a dinâmica do HIV com mutação
}

\author{
Claudia M. Dias ${ }^{1}$ \\ Programa de Pós Graduação em Modelagem Matemática e Computacional, Universidade Federal Rural \\ do Rio de Janeiro, Nova Iguaçu, RJ, Brasil \\ Dayse H. Pastore ${ }^{2}$ \\ Departamento de Matemática, Centro Federal de Educação Tecnológica, Rio de Janeiro, RJ, Brasil \\ Edilson F. Arruda ${ }^{3}$ \\ Department of Decision Analytics and Risk, Southampton Business School, University of Southampton, \\ Southampton, UK \\ Instituto Alberto Luiz Coimbra de Pós Graduação e Pesquisa de Engenharia, Universidade Federal do Rio \\ de Janeiro, Rio de Janeiro, RJ, Brasil
}

\begin{abstract}
Resumo. Este trabalho apresenta um modelo de equações diferenciais e integro-diferenciais para a dinâmica da infecção pelo vírus da imunodeficiência adquirida (HIV). Tais modelos levam em conta os processos de mutação que o vírus sofre depois de adentrar o sistema imunológico. O objetivo é acompanhar a evolução da dinâmica do vírus no sistema imunológico, uma vez que células de defesa (CTL) são recrutadas para combater exclusivamente cada uma das mutações. Como essas células não estarão disponíveis para combater diferentes cepas, é muito importante entender e modelar a evolução das cepas a fim de prevenir o colapso do sistema imunológico.
\end{abstract}

Palavras-chave. HIV, Modelagem Matemática, Mutação.

\section{Introdução}

Pela sua agressividade e pela forma com que ataca o organismo e elimina suas defesas, o vírus da imunodeficiência adquirida (HIV) vem intrigando cientistas desde a década de 80. Existe desde então um crescente interesse por modelos matemáticos para a dinâmica do vírus [2, 19]. Neste trabalho, estudamos sistemas diferenciais e íntegro-diferenciais que modelam a dinâmica do HIV no organismo humano, considerando a possibilidade de mutação. A validação é feita por meio de experimentos numéricos.

Pioneiros na área, Nowak e Bangham descreveram a dinâmica do HIV no organismo humano por meio de uma série de modelos de equações diferencias, considerando a interação do vírus com as células sadias e infectadas [19]. Os modelos foram expandidos posteriormente, introduzindo as células de defesa do organismo e, mais adiante, a mutação do vírus. A evolução pode ser constatada em $[1,11,20,29]$. Mais recentemente, o controle dos níveis de medicação foi incorporado a um modelo que contempla as interações entre o sistema imunológico e o HIV [22]. por ser o HIV um vírus de RNA altamente mutante, o presente trabalho incorpora a mutação do vírus a esse modelo. Consideramos ainda os resultados de Frid et al. [10], que utilizam um sistema íntegrodiferencial contínuo para modelar um sistema de quase-espécies produzidas por mutações sucessivas do vírus. É com base nesse paradigma que este trabalho propõe um modelo integro-diferencial para a dinâmica do HIV no organismo humano.

\footnotetext{
1 mazzaclaudia@gmail.com

2dpastore@cefet-rj.br

3 e.f.arruda@southampton.ac.uk
} 


\section{Modelagem da dinâmica no corpo humano}

Primeiramente, considera-se o modelo de Pastore et al. [22]:

$$
\left\{\begin{array}{l}
\dot{x}=\lambda_{x}-\mu_{x} x-\beta_{v} x v \\
\dot{y}=\beta_{v} x v-\mu_{y} y-p_{y} y z_{a} \\
\dot{v}=k_{v} \mu_{y} y-\mu_{v} v \\
\dot{z}=\lambda_{z}-\mu_{z} z-\beta_{z} z y \\
\dot{z_{a}}=\beta_{z} z y-\mu_{z} z_{a}
\end{array}\right.
$$

Nas equações acima, a variável $x$ representa células suscetíveis, com as quais o HIV pode se conectar. A variável $y$ representa as células já conectadas ao HIV (infectadas). Usa-se $v$ para representar o vírus livre presente no corpo e emprega-se a variável $z$ para descrever a população de células T assassinas. Finalmente, a variável $z_{a}$ representa a população de células CTL ativadas para combater as células infectadas, respondendo com anticorpos [6].

No sistema de equações em (1), $\mu_{x}$ é a taxa de mortalidade de células suscetíveis; $\mu_{y}$ a taxa de mortalidade de células infectadas; $\mu_{v}$ a taxa mortalidade do vírus; $\mu_{z}$ a taxa mortalidade de células CTL; $k_{v}$ é o número médio de vírus livres liberados por uma célula infectada; $\beta_{z}$ a taxa de ativação da resposta imune; $\beta_{v}$ a taxa de infecção pelo vírus; $p_{y}$ a taxa de destruição de células infectadas; $\lambda_{x}$ a taxa de fornecimento de células suscetíveis; e $\lambda_{z}$ a taxa de fornecimento de células CTL. O modelo não considera o controle da medicação. Ao se introduzir a ideia de que uma certa quantidade de diferentes tipos de vírus pode afetar o organismo, considera-se que $\beta_{i}$ é a taxa de infecção pelo vírus do tipo $i \in\{1, \ldots, n\}$. Assim, o modelo proposto simula, através de um operador integral, o processo de mutação viral.

\section{O modelo integro-diferencial}

O modelo com mutação pode ser escrito na forma integro-diferencial:

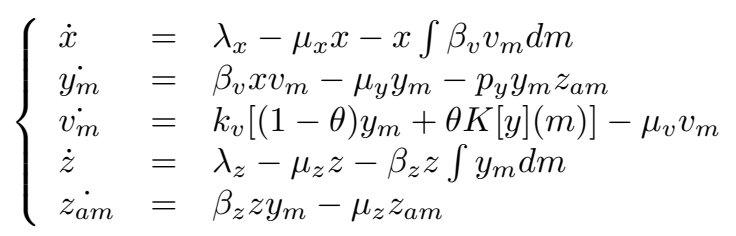

sendo $\theta \in[0,1]$ a probabilidade de mutação. As variáveis $y_{m}, v_{m}$ e $z_{a m}$ dependem do tempo $t \mathrm{e}$ do tipo de vírus $m \in \Omega$, espaço de mutação do vírus. Considera-se que $\Omega$ é uma espaço de medida $\sigma$-finita. Definimos o operador de mutação como o operador integral

$$
K[y](m)=\int_{\Omega} K\left(m, m^{\prime}\right) y\left(m^{\prime}\right) d m^{\prime},
$$

responsável pelo surgimento da mutação.

Desta forma, considerando-se que exista inicialmente um único tipo de vírus, o operador (3) fará com que surjam novos tipos de vírus, através da mutação do RNA do vírus no interior da célula infectada, desde que se considere o núcleo do operador positivo e pertencente a $L^{1}(\Omega \times \Omega)$. Observe que $\theta$ é a probabilidade de mutação e que $\theta=0$ reproduz o sistema original sem mutação.

\subsection{Resultados para o modelo com mutação}

A partir das condições iniciais em [22] e utilizando os parâmetros encontrados em [3,22], simulamos a dinâmica do HIV no corpo humano por um período de aproximadamente um ano. Assim, 


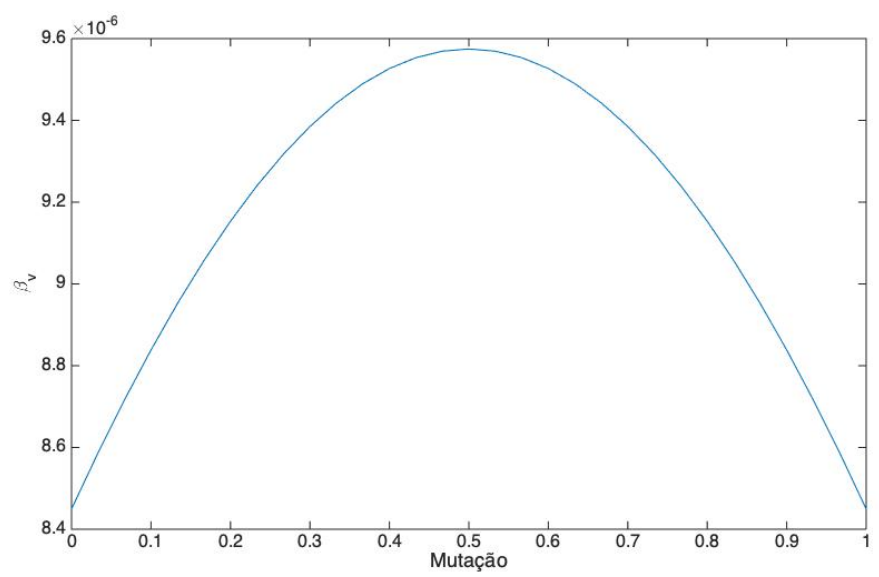

Figura 1: Função $\beta_{v}$.

$\beta_{v}=2,4 \times 10^{-5} \frac{e^{-\frac{1}{2}\left(\mu-\frac{1}{2}\right)^{2}}}{\sqrt{2 \pi}}$ e $K\left(\mu, \mu^{\prime}\right)=\frac{e^{-\frac{1}{2}\left(\mu-\frac{1}{2}\right)^{2}}}{\sqrt{2 \pi}}$. A Figura 1 apresenta a função $\beta_{v}$ utilizada nas simulações. Na Figura 2, vemos a seguinte sequência de soluções: população $x$ (células suscetíveis); população $z$ (células T assassinas); $\int y_{m}$ (soma de todas as células já conectadas ao HIV ou infectadas); uma visão global de $y_{m} ; \int z_{a m}$ (acumulado da população de células CTL ativadas para combater as células infectadas), visão global de $z_{a m}$; população de vírus livre; $\int v_{m}$ acumulado; e a visão global de $v_{m}$. As discretizações usadas para as simulações numéricas foram baseadas em [21]. Nota-se que o modelo aqui apresentado representa bem a dinâmica da infecção intracelular estando de acordo com os resultados encontrados em Arruda et al. [1], sem mutação. As células TCD 4 mantém um padrão normal considerando-se um paciente infectado sem tratamento com retro-terapia, enquanto que as células de defesa CTL caem bruscamente. Já a soma das células de defesa ativadas para o HIV crescem em grande escala conforme o vírus sofre mutação. Padrão este que é mantido durante a simulação. Observe que as cepas mais prevalentes ao longo do período são aquelas com maiores valores de $\beta_{m}$. Isso significa que as cepas que mais se reproduzem tendem a ser dominantes.

\section{Conclusões}

Uma vantagem desse modelo é que se pode observar o comportamento da infecção dependendo da mutação do vírus. Ainda se observa uma possível saturação do sistema imunológico por conta da quantidade de tipos diferentes de mutação. O surgimento de células de defesa CTL especificas para cada tipo de mutação gera uma grande quantidade de células de defesas ativadas para o HIV, o que pode levar o organismo a ficar indefeso ao ataque de outras infecções, já que estará voltado ao combate a várias cepas do HIV. O modelo permite assim uma importante análise sobre a saturação do sistema imunológico quando o indivíduo portador do HIV passa muitos anos sem tratamento.

O próximo passo do trabalho será a introdução da terapia retro-viral como mecanismo de controle do HIV, com vistas à observação do efeito da mutação no tratamento da doença. 

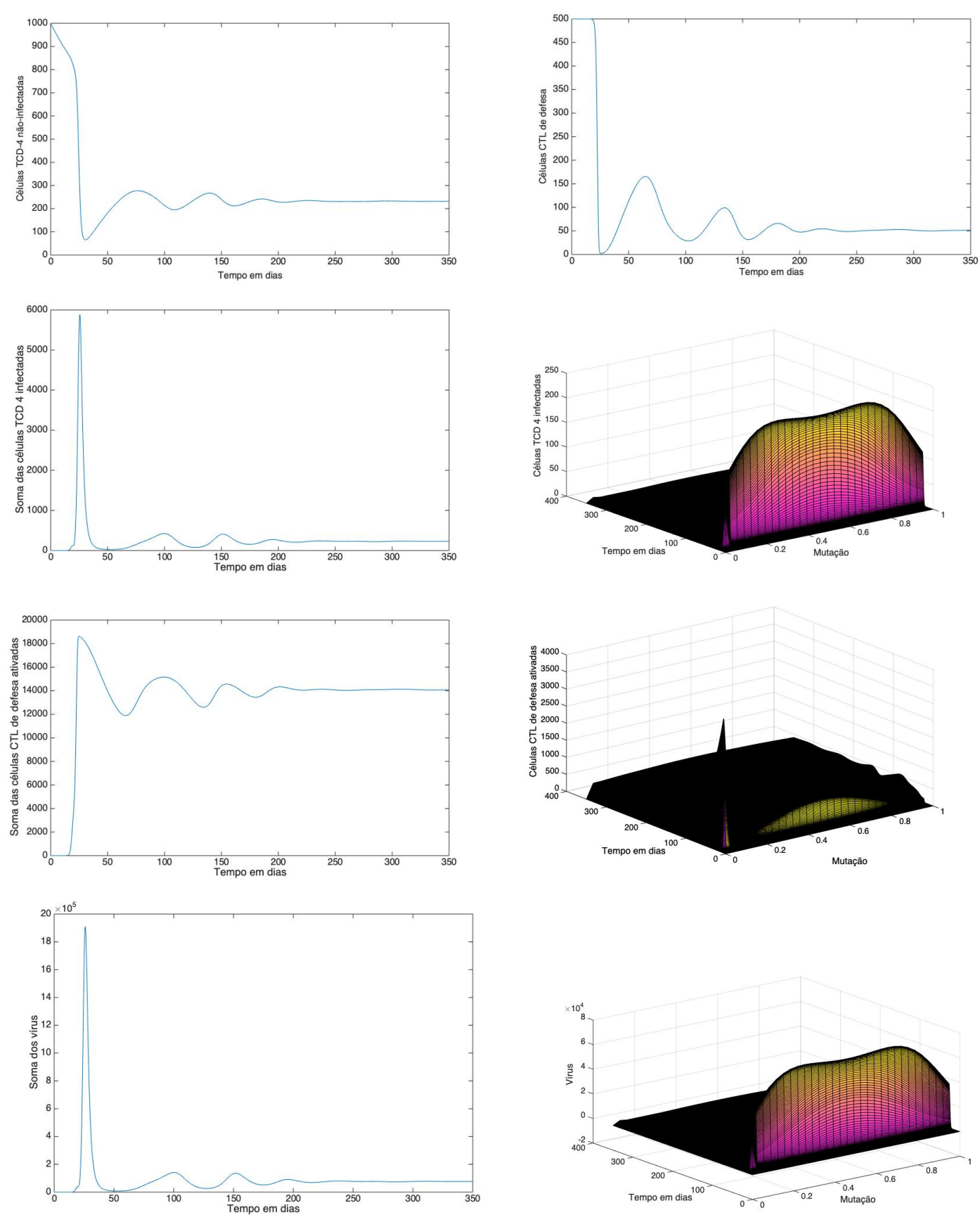

Figura 2: Resultado das simulações 


\section{Agradecimentos}

O presente trabalho foi realizado com apoio da Coordenação de Aperfeiçcoamento de Pessoal de Nível Superior Brasil. Brasil (CAPES). Código de Financiamento 001.

\section{Referências}

[1] Arruda, E. F., Dias, C. M., de Magalhães, C. V., Pastore, D. H., Thomé, R. C. A., Yang, H. M. An Optimal Control Approach to HIV Immunology. Applied Mathematics, 6, 1115-1130, 2015.

[2] Balamurali, M., Petravic, J., Loh, L., Alcantara, S., Kent, S. J., Davenport, M. P. Does cytolysis by CD8+ T cells drive immune escape in HIV infection?, The Journal of Immunology, 185 (9), 5093-5101, 2010.

[3] Bouin, E., Garnier, J., Henderson, C., Patout, F. Thin Front Limit of an Integro-differential Fisher-KPP Equation with Fat-Tailed Kernels. SIAM Journal on Mathematical Analysis, 50(3), 3365-3394. 2018. DOI: 10.1137/17M1132501

[4] Chun, T., Carruth, L., Finzi, D., Shen, X., Digiuseppe, J., Taylor, H., Hermankova, M., Chadwick, K., Margolick, J., Kuo, Y., Brookmeyer, R., Zeiger, M., Barditch-Crovo, P., Siliciano, R. Quantification of latente tissue reservoirs and total body viral load in HIV-1 infection. Nature, 387, 183-188, 1997.

[5] Daugas, E., Rougier, J. HAART-related nephropathies in HIV-infected patients. Kidney International, 67, 393-403, 2005.

[6] De Boer, R.J. Mathematical models of human CD4 T-cell population kinetics. Neth J Med, 60, 17-26, 2002.

[7] Dorf, R. C., Bishop, R. H.Modern Control Systems, 9th Edition. Prentice Hall, 2001.

[8] Eisele, E., Siliciano, R. Redefining the viral reservoirs that prevent HIV-1 eradication. Immunity, 37 (3), 377-388, 2012.

[9] Finzi, D., Blankson, J., Siliciano, J., Margolick, J., Chadwick, K., Pierson, T., Smith, K., Lisziewicz, J., Lori, F., Flexner, C., Quinn, T., Chaisson, R., Rosenberg, E., Walker, B., Gange, S., Gallant, J., Siliciano, R. Latent infection of CD4+ T cells provides a mechanism for lifelong persistence of HIV-1, even in patients on effective combination therapy. Nat Med, $5(5), 512-517,1999$.

[10] Frid, H., Jabin, P-E., Perthame, P. Global stability of steady solutons for a model in virus dynamics. ESAIM: Mathematical Modelling and Numerical Analysis, 37 (4) 709 -723, 2003.

[11] Grégio, J., Caetano, M., Yoneyama, T. State estimation and optimal long period clinical treatment of HIV seropositive patients. Anais da Academia Brasileira de Ciências, 81, 3-12, 2009.

[12] Ho, P., Neumann, A., Perelson, A., Chew, W., Leonardo, J., Markowitz, M. Rapid turnover of plasma virions and CD4 lymphocytes in HIV-1 infection. Nature, 373, 123-126, 1995.

[13] Kilby, J. M, Goepfert, P. A., Miller, A. P., Gnann Jr, J. W., Sillers, M., Saag, M. S., Bucy, R. P. Recurrence of the acute HIV syndrome after interruption of antiretroviral therapy in a patient with chronic HIV infection: a case report. Ann Intern Med, 133, 435-438, 2000. DOI: 10.7326/0003-4819-133-6. 
[14] Kirk, D. Optimal control theory: An introduction. Prentice-Hall, 1970.

[15] Kreysig, E. Introductory Functional Analysis with Applications. Wiley, 1978.

[16] Lafeuillade, A., Poggi, C., Profizi, N. Human immunodeficiency virus type 1 in lymph nodes compared with plasma. J.Infect. Dis., 174, 404-407, 1996.

[17] Lewis, F., Syrmos, V. Optimal Control. John Wiley and Sons, 1995.

[18] Mclean, A. Infectious disease modeling. In: Kanki, P., Grmes, D. J. (Eds.), Infectious Diseases. Springer, New York, 99-115, 2013.

[19] Nowak, M., Bangham, C. Population dynamics of immune responses to persistent viruses. Science, 272 (5258), 74-79, 1996.

[20] Nowak, M., May, R. Virus dynamics: Mathematical principles of immunology and virology. Oxford University Press, Oxford, 2000.

[21] Pastore, D. On the numerical simulation of a class of HIV models. Mathematical and Computer Modelling, 47 (7), 2008.

[22] Pastore, D. H., Thomé, R. C. A., Dias, C. M., Arruda, E. F., Yang, H. M. A model for interactions between immune cells and HIV considering drug treatments. Comp. Appl. Math., 37 (1), 282-295, 2018.

[23] Perelson, A. Modelling viral and immune system dynamics. Nat Rev Immunol, 2, 28-36, 2002.

[24] Perelson, A., Nelson, P. Mathematical analysis of HIV-1 dynamics in vivo. SIAM Rev., 41 (1), 3-44, 1999.

[25] Perelson, A., Ribeiro, R. Modeling the within-host dynamics of HIV infection. BMC Biology, $11(1), 96,2013$.

[26] Pontryagin, L., Boltyanskij, V., GamKrelidze, R., Mishchenko, E. The Mathematical Theory of Optimal Processes. Interscience Publishers, New York, 1961.

[27] Siewe, B., Wallace, J., Rygielski, S., Stapleton, J. T., Martin, J., Deeks, S. G., Landay, A. Regulatory B cells inhibit cytotoxic t lymphocyte (CTL) activity and elimination of infected CD4 T cells after in vitro reactivation of HIV latent reservoirs. PLoS ONE, 9 (4), 2014.

[28] Sreenivasan, S., Dasegowda, V. Adverse effects after HAART initiation in resource-limited settings: a prospective study from Mysore, India. J Infect Dev Ctries, 4 (11), 750-753, 2010.

[29] Shu, H., Wang, L., Watmough, J. Sustained and transient oscillations and chaos induced by delayed antiviral immune response in an immunosuppressive infection model. J Math Biol, 68, 477-503, 2014.

[30] Torres, R., Lewis, W. Aging and HIV/AIDS: pathogenetic role of therapeutic side effects. Laboratory Investigation, 94, 120-128, 2014. 